\title{
Analyzed the Resistance of Influenza B Virus in Shangrao
}

\author{
Changhe Cheng ${ }^{1, *}$, Lanxing Liu ${ }^{2}$, Xiaolong Liu ${ }^{1}$, Zhang Yanyan ${ }^{3}$, Lai Yu ${ }^{1}$ \\ ${ }^{1}$ Department of Pharmacology, Jiangxi Medical College, Shangrao, P. R. China \\ ${ }^{2}$ Department of Party History and Party Construction, The Party School of the CPC Shangrao Municipal Committee, Shangrao, P. R. China \\ ${ }^{3}$ Clinical Laboratory, Shangrao Center for Disease Control and Prevention, Shangrao, P. R. China
}

\section{Email address:}

chengchanghe@hotmail.com (Changhe Cheng)

${ }^{*}$ Corresponding author

\section{To cite this article:}

Changhe Cheng, Lanxing Liu, Xiaolong Liu, Zhang Yanyan, Lai Yu. Analyzed the Resistance of Influenza B Virus in Shangrao. Biomedical Sciences. Vol. 7, No. 3, 2021, pp. 95-98. doi: 10.11648/j.bs.20210703.16

Received: September 4, 2021; Accepted: September 22, 2021; Published: September 30, 2021

\begin{abstract}
Influenza B is one of the main pathogens of human infection, especially among children and adolescents. The incidence rate and mortality rate are higher after infection. Vaccines and drugs are the most effective means to prevent and treat influenza virus. However, due to the characteristics of easy mutation of influenza virus, there are many drug-resistant strains in clinical using. In this study, we analyzed the molecular characterization of neuraminidase (NA) genes and NA drug resistance of influenza B virus in Shangrao during 2019 years. The specimens of nasopharyngeal swabs were collected from influenza cases of monitor hospitals, and then the collection of 10 strains of influenza B virus were randomly selected for detection by MDCK, positive strains were identified by hemagglutination test (HA) and hemagglutination inhibition test (HI). And virus RNA were extracted with QIAamp Viral RNA Mini Kit. Fragments of NA genes were amplified by one-step RT-PCR and then were sequenced. The data obtained were analyzed with the software DNAStar 6.0 and Mage 5.0. We observed that the nucleotides of NA gene of 10 strains had no mutation in catalytic residues and framework residues of NA gene. And strongly suggest that all viruses were sensitive to neuraminidase inhibitors, however continuous resistance surveillance is necessary for control and prevention influenza.
\end{abstract}

Keywords: Influenza B Virus, Resistance, NA Gene

\section{Introduction}

Influenza virus could be divided into four types according to the antigenicity of nucleoprotein (NP) and matrix protein (M1) [1, 2]. Influenza $B$ is the main pathogen of human infection. Although most cases do not cause worldwide pandemic, IBV induced seasonal influenza wasn't to be ignored. The incidence rate and mortality rate of infection were high, especially with children and adolescents [3-5]. In the long-term evolution, IBV could be divided into two main lineages according to the nucleotide sequence difference of HA fragment, namely $\mathrm{B}$-victoria branch represented by $\mathrm{B} / \mathrm{Victoria} / 2 / 87$ strain and B-yamagata branch represented by B/ Yamagata/ 16/88 strain [6, 7]. The neuraminidase inhibitor was main clinical treated. With the long-term use of this drug, the drug-resistant was serious [8]. This report was study the gene characteristics of neuraminidase of influenza B virus in our city, analyzed the drug resistance of influenza B virus, for clinical medication and disease control.

\section{Data and Methods}

\subsection{Sample Collected}

Throat swab samples of suspected influenza patients collected from Shangrao sentinel hospital were inoculated into MDCK cells for virus isolation. Positive strains were identified by hemagglutination test (HA) and hemagglutination inhibition test (HI). According to the sampling time, seven influenza B virus strains were randomly selected (all strains were reviewed and identified by the national influenza Center).

\subsection{Virus Extraction and Amplification}

Extracted RNA of the above 10 selected influenza B virus strains with QIAamp Viral RNA Mini Kit (Qiagen company, Germany); and adding primers (NA-5' upper primer 
GCAGAAGCAGAGCATATTCTTAG, lower primer ATGGACAAATCCTCCCTTGATGC; NA-3' upper primer GCACTCCTAATTAGCCCTCATAGA, lower primer AGGACAATTGTTCAAAC) for RT-PCR (gene amplification reaction conditions: $60^{\circ} \mathrm{C}, 1 \mathrm{~min} ; 42^{\circ} \mathrm{C}, 10 \mathrm{~min}$; $\left.50^{\circ} \mathrm{C}, 30 \mathrm{~min} ; 95^{\circ} \mathrm{C}, 15 \mathrm{~min}\right)$. The PCR cycle was as follows: $94^{\circ} \mathrm{C}, 40 \mathrm{~s} ; 50^{\circ} \mathrm{C}, 40 \mathrm{~s} ; 72^{\circ} \mathrm{C}, 1 \mathrm{~min}, 30 \mathrm{~s} ; 35$ cycles, $72^{\circ} \mathrm{C}, 10$ min.) with Qiagen one step RT-PCR kit.

\subsection{Nucleotide Sequence}

Sequenced PCR producted by Shanghai Shenggong Bioengineering Technology Service Co., Ltd.

\subsection{Analyzed Sequence}

Analyzed the nucleotide sequence with Dnastar6.0 and mage5.0 and compared with the NA gene sequence of B/Beijing/1/1987 (designated by National Influenza Center) NO: M54967.

\section{Results}

\subsection{Genetic Evolution Analysis}

The 10 selected strains of influenza B virus in Shangrao City were Yamagata strains. The NO. as follows: B/jiangxishangrao/1019/2019, B/jiangxishangrao/1041/2019, B/jiangxishangrao/1117/2019, B/jiangxishangrao/1056/2019, $\mathrm{B} /$ jiangxishangrao/1229/2019, B/jiangxishangrao/1252/2019, $\mathrm{B} / \mathrm{jiangxishangrao} / 1332 / 2019$, B/jiangxishangrao/1339/2019 B/jiangxishangrao/1343/2019, B/jiangxishangrao/1347/2019.
10 strains were in the same branch compared with B/Beijing/1/1987, while B/jiangxishangrao/1117/2019 and $\mathrm{B} /$ jiangxishangrao/1139/2019 were slightly different from the other 8 strains, so there were a small branch. (Figure 1)

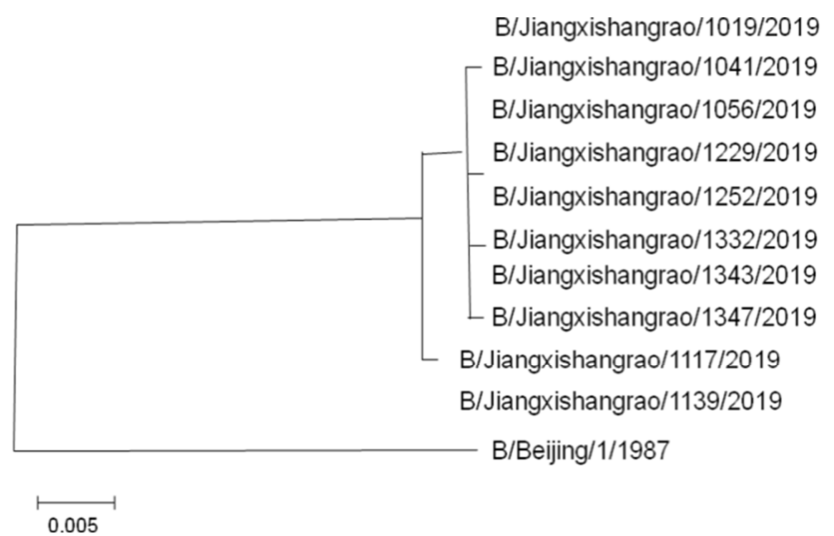

Figure 1. Phylogenetic tree of NA gene of influenza B virus detected Shangrao city.

\subsection{Analyzed Nucleotide and Amino Acid Homology}

Compared with the sequences of B/Beijing/1/1987, the nucleotide homology was $93.4 \%-94.1 \%$, the amino acid homology was $92.1 \%-92.9 \%$, and the nucleotide sequence homology of NA gene was $99.1 \%-100 \%$, the amino acid sequence homology was $99.3 \%-100 \%$ (Tables 1 and 2 ).

Table 1. Analyzed of nucleotide homology of NA gene in 10 influenza B viruses (\%).

\begin{tabular}{|c|c|c|c|c|c|c|c|c|c|c|c|}
\hline $\begin{array}{ll}\text { nucleotide homology } & \text { NO. stain } \\
\end{array}$ & (1) & (2) & (3) & (4) & (5) & (6) & (7) & (8) & (9) & (10) & (11) \\
\hline$(1)$ & $*$ & 93.6 & 93.4 & 93.5 & 93.5 & 93.7 & 93.5 & 93.5 & 94.0 & 94.1 & 93.8 \\
\hline (2) & 7.0 & $*$ & 100 & 99.4 & 99.8 & 99.8 & 99.9 & 100 & 99.5 & 99.6 & 99.6 \\
\hline (3) & 7.1 & 0 & $*$ & 99.5 & 99.8 & 99.8 & 100 & 99.6 & 99.7 & 99.6 & 100 \\
\hline (4) & 6.8 & 0.4 & 0.4 & $*$ & 99.4 & 99.5 & 99.6 & 99.6 & 99.5 & 99.5 & 99.9 \\
\hline (5) & 7.0 & 0.1 & 0.1 & 0.6 & $*$ & 99.8 & 100 & 99.9 & 99.5 & 99.4 & 100 \\
\hline (6) & 7.0 & 0.1 & 0.1 & 0.5 & 0.2 & $*$ & 99.9 & 99.9 & 99.4 & 100 & 99.4 \\
\hline (7) & 6.9 & 0 & 0 & 0.4 & 0.1 & 0.1 & $*$ & 100 & 100 & 99.5 & 99.4 \\
\hline (8) & 6.8 & 0 & 0 & 0.4 & 0.1 & 0.1 & 0 & $*$ & 99.6 & 99.6 & 99.9 \\
\hline (9) & 7.0 & 0.1 & 0.1 & 0 & 0 & 0.3 & 0.3 & 0 & $*$ & 100 & 99.8 \\
\hline (10) & 6.9 & 0 & 0 & 0.2 & 0.2 & 0.1 & 0.1 & 0 & 0 & $*$ & 100 \\
\hline (11) & 7.1 & 0 & 0 & 0.4 & 0.2 & 0.2 & 0 & 0 & 0.1 & 0.1 & $*$ \\
\hline
\end{tabular}

(1) B/beijing/1/1987, (2) B/jiangxishangrao/1019/2019, (3) B/jiangxishangrao/1041/2019, (4) B/jiangxishangrao/1117/2019, (5) B/jiangxishangrao/1056/2019, (6) B/jiangxishangrao/1229/2019, (7) B/jiangxishangrao/1252/2019, (8) B/jiangxishangrao/1332/2019, (9) B/jiangxishangrao/1339/2019, (10) B/jiangxishangrao/1343/2019, (11) B/jiangxishangrao/1347/2019.

Table 2. Analyzed of amino acid homology of NA gene in 10 influenza B viruses (\%).

\begin{tabular}{|c|c|c|c|c|c|c|c|c|c|c|c|}
\hline $\begin{array}{ll}\text { nucleotide homology } & \text { NO. stain } \\
\end{array}$ & (1) & (2) & (3) & (4) & (5) & (6) & (7) & (8) & (9) & (10) & (11) \\
\hline$(1)$ & $*$ & 92.5 & 92.1 & 92.4 & 92.4 & 92.6 & 92.5 & 92.4 & 92.9 & 92.8 & 92.2 \\
\hline (2) & 7.7 & $*$ & 100 & 99.6 & 99.8 & 99.7 & 99.7 & 100 & 100 & 99.5 & 99.5 \\
\hline (3) & 7.6 & 0 & $*$ & 99.6 & 99.8 & 99.8 & 100 & 100 & 99.4 & 99.6 & 100 \\
\hline (4) & 7.7 & 0.4 & 0.4 & $*$ & 99.4 & 99.6 & 99.6 & 99.6 & 99.4 & 99.7 & 100 \\
\hline (5) & 7.7 & 0.2 & 0.2 & 0.6 & $*$ & 99.3 & 99.5 & 99.8 & 99.5 & 99.3 & 100 \\
\hline (6) & 7.6 & 0 & 0 & 0.4 & 0.2 & $*$ & 100 & 100 & 99.6 & 99.5 & 99.9 \\
\hline (7) & 7.7 & 0 & 0 & 0.4 & 0.2 & 0 & $*$ & 100 & 99.6 & 100 & 99.9 \\
\hline (8) & 7.7 & 0 & 0 & 0.4 & 0.2 & 0 & 0 & $*$ & 100 & 100 & 99.6 \\
\hline
\end{tabular}




\begin{tabular}{|c|c|c|c|c|c|c|c|c|c|c|c|}
\hline $\begin{array}{ll}\text { nucleotide homology } & \text { NO. stain } \\
\end{array}$ & (1) & (2) & (3) & (4) & (5) & (6) & (7) & (8) & (9) & (10) & (11) \\
\hline$(9)$ & 7.7 & 0 & 0 & 0.3 & 0.3 & 0.1 & 0.1 & 0 & * & 99.9 & 100 \\
\hline (10) & 7.7 & 0.1 & 0 & 0.2 & 0.2 & 0 & 0 & 0 & 0 & $*$ & 99.7 \\
\hline (11) & 7.6 & 0 & 0.2 & 0.3 & 0.3 & 0 & 0 & 0.1 & 0.1 & 0.1 & $*$ \\
\hline
\end{tabular}

(1) B/beijing/1/1987, (2) B/jiangxishangrao/1019/2019, (3) B/jiangxishangrao/1041/2019, (4) B/jiangxishangrao/1117/2019, (5) B/jiangxishangrao/1056/2019, (6) B/jiangxishangrao/1229/2019, (7) B/jiangxishangrao/1252/2019, (8) B/jiangxishangrao/1332/2019, (9) B/jiangxishangrao/1339/2019, (10) B/jiangxishangrao/1343/2019, (11) B/jiangxishangrao/1347/2019.

\subsection{Analyzed of NA Gene Resistance Loci}

According to the nucleotide sequence, the top 500 amino acid sequences of NA gene were deduced, among which the b-numbering (R-116, D-149, R-150, r-223, e-275, r-292 There were no substitutions of amino acids and auxiliary sites (e-117 r-154, w-177, s-178, d-197, i-221, e-226, h-273, e-276 and $\mathrm{n}-294$, e428) in the 10 strains, which indicated that the resistance of NA gene had not changed.

\section{Discussion}

The main mechanism of mutation evolution of influenza B virus was gene fragment rearrangement and base insertion or deletion, which couldn't be occur only between Victoria and Yamagata strains, but also between viruses of different branches of the same strain [9-10]. Although the Victoria and Yamagata strains of influenza B have different evolutionary characteristics, they have the characteristics of coexistence and coevolution in the population [11]. Influenza virus neuraminidase (NA) was an important surface glycoprotein of influenza virus, which has antigenicity and can catalyze the hydrolysis of sialic acid to help mature influenza virus escape from host cells and infect new cells [12]. NA can change the virulence and drug resistance of viruses through antigenicity transformation. When viruses mutate to make them more conducive to survival or escape host immune response, new strains will replace old strains, leading to virus evolution [13]. Through sequencing analysis of NA Gene of 10 strains of influenza $B$ virus, the results showed that the difference between 10 strains and standard strain B/Beijing/1/1987 was very small from three aspects of phylogenetic tree, nucleotide and amino acid homology, and they were in the same branch on the phylogenetic tree; the nucleotide and amino acid homology were more than $90 \%$; from the amino acid sequence, the gene sites affecting drug resistance The results indicated that the NA Gene of influenza B virus had not mutated.

Neuraminidase inhibitors (NAIS) are the only drugs for the treatment of influenza B. influenza virus neuraminidase is used as a drug target. Genome wide analysis showed that the amino acids of b-numbering (R-116, D-149, R-150, r-223, $\mathrm{e}-275, \mathrm{r}-292, \mathrm{r} 374$ and 406) and auxiliary sites (e-117, r-154, w-177, s-178, d-197, i-221, e-226, h-273, e-276 and n-294, e428) of NA gene were the main factors affecting the drug resistance [14]. The results of this study showed that none of these sites affected drug resistance, indicating that influenza virus is sensitive to neuraminidase inhibitors. This is also consistent with the drug resistance gene analysis of influenza
B virus reported by Yan yan Zhang [15]. In the city of shangrao Jiangxi Province from 2014 to 2017 indicating that there is no drug resistance of influenza B virus in this region so far. In addition, it has been reported that NA gene mutation at 198, 222, 250, 402 can reduce the sensitivity of neuraminidase inhibitors [16]. This experiment also found that except B/jiangxishangrao/1166/2019, the other nine strains had a402t substitution at amino acid 402. These substitution sites indicated that the variation of influenza $B$ may have affected the sensitivity of neuraminidase inhibitors, but the specific impact on clinical use of drugs, especially the impact of drug resistance, still needs further study.

\section{Conclusion}

In summary, our studies provide evidence that he nucleotides of NA gene of 10 strains had no mutation in catalytic residues and framework residues of NA gene, all viruses were sensitive to neuraminidase inhibitors in Shangrao during 2019.

\section{Conflict of Interest}

All authors of the "Analyzed the resistance of influenza B virus in Shangrao" manuscript have directly participated in planning, execution, and analysis. The contents of this manuscript have not been copyrighted or published previously. The contents of this manuscript are not now under consideration for published elsewhere. There are no directly related manuscript or abstracts, published or unpublished, by any authors of this manuscript.

\section{Acknowledgements}

This work was supported by grants from Jiangxi Provincial Department of Education Science and technology project (No. 20170432).

\section{References}

[1] Caini S, Kusznierz G, Garate V V, et al. The epidemiological signature of influenza $\mathrm{B}$ virus and its $\mathrm{B} /$ Victoria and $\mathrm{B} /$ Yamagata lineages in the 21st century. PLoS ONE 2019. 14 (9), 1-17.

[2] Cassini A, Colzani E, Pini A, et al. Impact of infectious diseases on population health using incidence-based disability-adjusted life years (DALYs): results from the Burden of Communicable Diseases in Europe study, European Union and European Economic Area countries, 2009 to 2013. Euro Surveill. 2018; 23 (16). 
[3] Zaraket H, Hurt AC. Clinch Barry et al. Burden of influenza B virus infection and considerations for clinical management. Antiviral Research, 2021, 185: 373-381.

[4] Jessica T, Guha A A, Florian K. Universal influenza virus vaccines and therapeutics: where do we stand with influenza $B$ virus. Current Opinion in Immunology, 2018, 53: 45-50.

[5] Owusu D, Hand J, Tenforde MW, et al. Early season pediatric influenza B/victoria virus infections associated with a recently emerged virus subclade-Louisiana, 2019. MMWR Morb Mortal Wkly Rep2020; 69: 40-3.

[6] Jennings L, Huang QS, Barr I, et al. Literature review of the epidemiology of influenza B disease in 15 countries in the Asia-Pacific region. Influenza Other Respir Viruses. 2018; 12 (3): $383-411$

[7] Skowronski DM, Hottes TS, De Serres G, et al. Influenza $\mathrm{B} / \mathrm{Victoria}$ antigen induces strong recall of $\mathrm{B} /$ Yamagata but lower B/Victoria response in children primed with two doses of B/Yamagata. Pediatr Infect Dis J. 2011; 30 (10): 833-839.

[8] Doyle JD, Campbell AP. Pediatric influenza and illness severity: what is known and what questions remain? Curr Opin Pediatr 2019; 31: 119-26.

[9] Del Mar C, Collignon P. How can we prepare better for influenza epidemics? BMJ, 2017, 359: j5007.
[10] Xie H, Xiang R, Wan HJ, et al. Reduced Influenza B-specific Post-Vaccination Antibody Cross-reactivity in the B/Victoria Lineage Predominant 2019/20 Season. Clin Infect Dis, 2020, 72 (11): e776-e783.

[11] Ivan K, Jonathan W. Yewdell. Influenza Hemagglutinin and Neuraminidase: Yin-Yang Proteins co-evolving to Thwart Immunity. Viruses, 2019, 11 (4): 346.

[12] Danielle LL, Katherine MR, Howard HC et a1. Estimates of global seasonal influenza-associated respiratory mortality: a modelling study. Lancet. 2018; 391 (10127): 1285-1300.

[13] Hang WJ, Li XY, Tan MJ, et al. Sisceptibility of inluenze B viruses to neuraminidase inhibitors isolated during 2013-2014 influenza season in mainland China. Chinese Journal of Virology. 2015, 6 (2): 152-156.

[14] Zhang YY, Lai Y, Xiong Y, et al. Neuraminidase gene profiling of influenza B virus in Shangrao. Chin J Chengmther, 2017, 17 (5): 538-540.

[15] Han GG, Li Y, Jiang CX, et al, Epidemiological characteristics of influenza and characteristics of hemagglutinin gene of B-Victoria strains in Hebei Province from 2018 to 2019. Chin J Prev Med, 2021, 55 (07): 847-852.

[16] Andrew J. Burnham, Tatiana Baranovich, et al. Neuraminidase inhibitors for influenza B virus infection. Antiviral Research, 2013, 100 (2): 520-534. 